OPEN ACCESS

Edited by:

Om Prakash,

National Centre for Cell Science, India

Reviewed by:

Xuehuan Feng,

University of Nebraska-Lincoln,

United States

Pan Liao,

Purdue University, United States

*Correspondence:

Chengjian Jiang

jiangcj0520@vip.163.com

Specialty section:

This article was submitted to

Evolutionary and Genomic

Microbiology,

a section of the journa

Frontiers in Genetics

Received: 20 October 2021 Accepted: 24 December 2021

Published: 13 January 2022

Citation:

Sun $H$, Cai $X$, Yan B, Bai $H$, Meng D, Mo X, He S, Su G and Jiang C (2022)

Multi-Omics Analysis of Lipid Metabolism for a Marine Probiotic Meyerozyma guilliermondii GXDK6

Under High $\mathrm{NaCl}$ Stress.

Front. Genet. 12:798535.

doi: 10.3389/fgene.2021.798535

\section{Multi-Omics Analysis of Lipid Metabolism for a Marine Probiotic Meyerozyma guilliermondii GXDK6 Under High $\mathrm{NaCl}$ Stress}

\author{
Huijie Sun ${ }^{1}$, Xinghua Cai ${ }^{1}$, Bing Yan ${ }^{2}$, Huashan Bai ${ }^{1}$, Duotao Meng ${ }^{1}$, Xueyan $\mathrm{Mo}^{1}$, Sheng $\mathrm{He}^{3}$, \\ Guijiao Su ${ }^{1}$ and Chengjian Jiang ${ }^{1,2 *}$ \\ ${ }^{1}$ State Key Laboratory for Conservation and Utilization of Subtropical Agro-bioresources, Guangxi Research Center for Microbial \\ and Enzyme Engineering Technology, College of Life Science and Technology, Guangxi University, Nanning, China, ${ }^{2}$ Guangxi Key \\ Lab of Mangrove Conservation and Utilization, Guangxi Mangrove Research Center, Guangxi Academy of Sciences, Beihai, \\ China, ${ }^{3}$ Guangxi Birth Defects Prevention and Control Institute, Maternal and Child Health Hospital of Guangxi Zhuang \\ Autonomous Region, Nanning, China.
}

Investigating microbial lipid regulation contributes to understanding the lipid-dependent signal transduction process of cells and helps to improve the sensitivity of microorganisms to environmental factors by interfering with lipid metabolism, thus beneficial for constructing advanced cell factories of novel molecular drugs. Integrated omics technology was used to systematically reveal the lipid metabolism mechanism of a marine Meyerozyma guilliermondii GXDK6 under high $\mathrm{NaCl}$ stress and test the sensitivity of GXDK6 to antibiotics when its lipid metabolism transformed. The omics data showed that when GXDK6 perceived $10 \% \mathrm{NaCl}$ stress, the expression of $A Y R 1$ and $\mathrm{NADPH}$-dependent 1-acyldihydroxyacetone phosphate reductase was inhibited, which weaken the budding and proliferation of cell membranes. This finding was further validated by decreased $64.39 \%$ of $\mathrm{OD}_{600}$ under $10 \% \mathrm{NaCl}$ stress when compared with salt-free stress. In addition, salt stress promoted a large intracellular accumulation of glycerol, which was also verified by exogenous addition of glycerol. Moreover, $\mathrm{NaCl}$ stress remarkably inhibited the expression of drug target proteins (such as lanosterol 14-alpha demethylase), thereby increasing sensitivity to fluconazole. This study provided new insights into the molecular mechanism involved in the regulation of lipid metabolism in Meyerozyma guilliermondii strain and contributed to developing new methods to improve the effectiveness of killing fungi with lower antibiotics.

Keywords: Meyerozyma guilliermondii, lipid metabolism, integrated omics technology, signal transduction, antibiotics

\section{INTRODUCTION}

Microbial lipid metabolism plays a key role in cell signaling, osmotic pressure balance, and the construction of cell membrane structures (Wang S. et al., 2021; Klug and Daum, 2014). Interfering with the lipid metabolism of microorganisms could significantly change their sensitivity to the environment and transform their life activities, thereby contributing to the targeted development of new microbial cell factories (Ferreira et al., 2018). The lipid metabolism in the Kyoto Encyclopedia of Genes and Genomes (KEGG) database is currently known to have 17 branch pathways. Among 


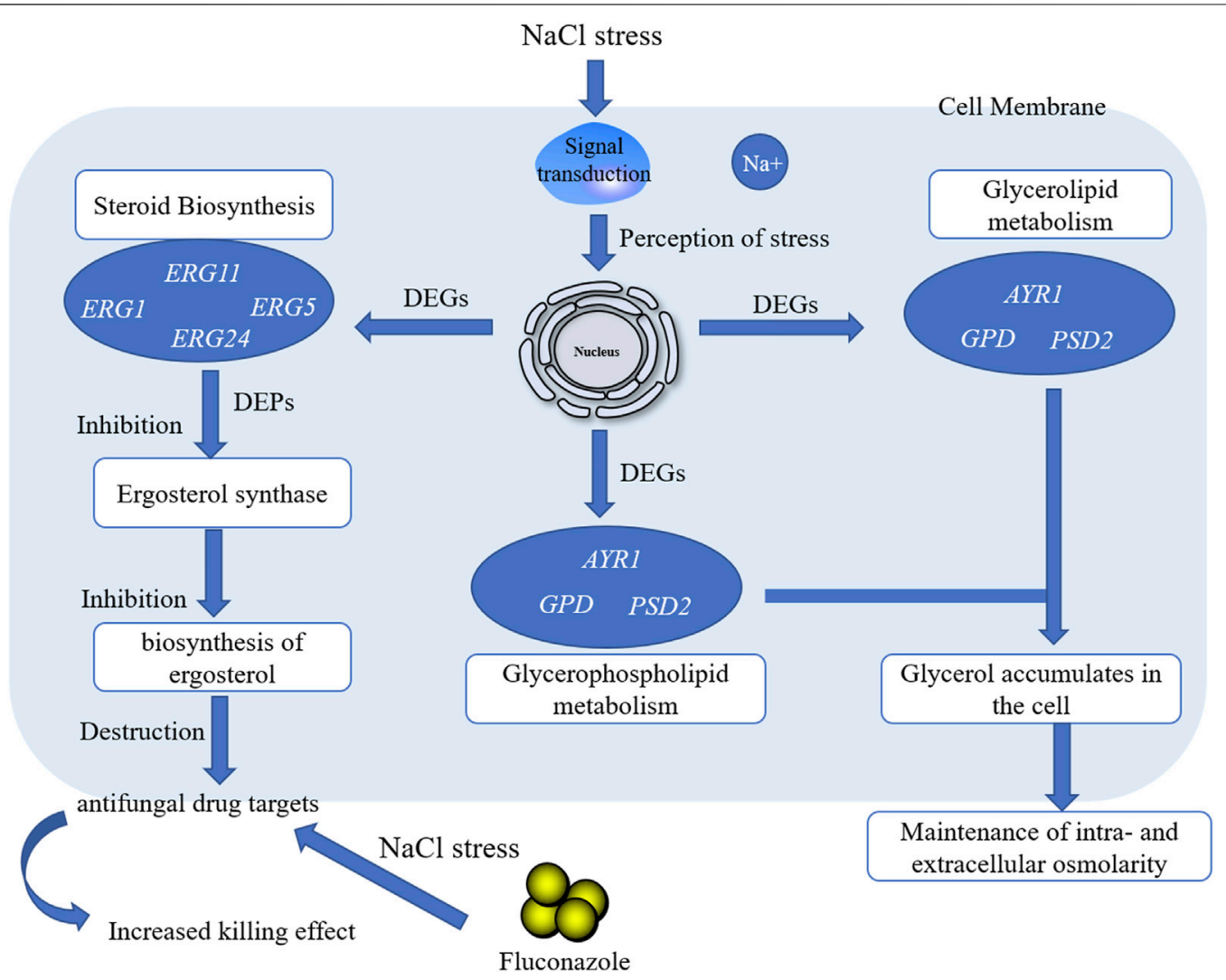

GRAPHICAL ABSTRACT |

them, steroid biosynthesis and sphingolipid metabolism are relevant to the microorganisms' cytoskeleton, which regulates their membrane fluidity and permeability (Kraft, 2017; Xu and Li, 2020). Glycerolipid metabolism is closed related to the construction of cell membrane structure (phospholipids and other lipids, varying by organelle type) and energy storage (primarily in the form of triacylglycerol) (Zhang and Reue, 2017). Glycerophospholipid metabolism is mainly linked to cell growth and proliferation (Nebauer et al., 2004). In addition, fatty acid metabolism (such as fatty acid biosynthesis, fatty acid degradation, fatty acid elongation, and biosynthesis of unsaturated fatty acids) are closely related to microbial cell signal transduction, and they are widely considered to be responsible for participating in and responding to extracellular environmental signal factors (Ross et al., 2003; Fan et al., 2016). These pathways of lipid metabolism are known to be indispensable for the basic life activities of microorganisms by supporting microbes to make precise physiological responses in different environments. Therefore, the lipid metabolism of microorganisms is important to forming the cytoskeleton and responding to environmental signal factors, thus enabling microorganisms to exhibit unique life activities in different environments.

The microbial lipid metabolism mechanism has gained increasing interest. Guo et al. reported that when
Saccharomyces cerevisiae was stressed by strong acid, its lipid products were remodeled, which was helpful for improving cell viability under acid stress (Guo et al., 2018). Randez-Gil et al. demonstrated that lipid metabolism was involved in regulating the composition of cell membranes in $S$. cerevisiae under lowtemperature conditions (Randez-Gil et al., 2018); Zhou et al. engineered $S$. cerevisiae to produce L-alkenes by manipulating fatty acid metabolism, enzyme selection, and the electron transfer system and expressing a transporter (Zhou et al., 2018). Therefore, the regulation of lipid metabolism is a useful method for microorganisms to enhance their survival ability under different adverse environments. However, few reports on the mechanism of lipid metabolism focused on how the lipids work and/or how this mechanism contributed to cells (Casanovas et al., 2015; Wang et al., 2018; Lu et al., 2021). A global regulatory network of lipid metabolism has not been revealed yet, resulting in random transformation, which is not conducive to accurately constructing advanced cell factories (Galafassi et al., 2015; Qi et al., 2017; Tian et al., 2019; Li C. et al., 2021). Therefore, mapping the lipid regulation network of microorganisms is an important prerequisite for constructing advanced cell factories by interfering with lipid metabolism.

In the previous study, a marine Meyerozyma guilliermondii GXDK6 that contained abundant lipid metabolism genes was obtained from subtropical marine mangrove microorganisms, 
and it showed excellent salt-tolerant survivability $(14 \% \mathrm{NaCl}$ and $18 \% \mathrm{KCl}$ ) (Mo et al., 2021). On the basis of its typical physicochemical characteristics, GXDK6 was hypothesized to have a unique regulatory network of lipid metabolism. GXDK6 can precisely regulate the expression of genes and/or proteins related to lipid metabolism to support its survival under salt stress conditions, which lead to greater sensitivity to certain environmental factors. The lipid regulation mechanism of GXDK6 under high-salt stress was systematically investigated by using an integrative omics technology (whole-genomic, transcriptomic, and proteomic) to test these hypotheses. The systematic analysis of the regulation network of lipid metabolism in $M$. guilliermondii strain provide a feasible reference for the construction of lipid cell factories.

\section{MATERIALS AND METHODS}

\section{Experimental Strain}

A yeast M. guilliermondii GXDK6 was obtained from the subtropical marine mangrove sediments, and was released by China General Microbiological Culture Collection Center (CGMCC) with the preservation number CGMCC No. 16007.

\section{Effect of $\mathrm{NaCl}$ Stress on the Survival of $M$. guilliermondii Strain}

GXDK6 was cultured at different $\mathrm{NaCl}$ concentrations $(0,5$, and $10 \%$ ) with $37^{\circ} \mathrm{C}$ and $200 \mathrm{rpm}$ for $0-48 \mathrm{~h}$, respectively. Its growth curve was evaluated by turbidimetric method. The cells were collected by centrifugation at $12,000 \mathrm{rpm}$ for $10 \mathrm{~min}$ and then washed three times with sterile saline $(0,5$, and $10 \% \mathrm{NaCl})$. The GXDK6 was further prepared into cell suspension and observed using scanning electron microscopy (SEM, Thermoelectric Company, American) to explore the influence of $\mathrm{NaCl}$ stress on the morphology.

\section{Whole-Genome Analysis for Lipid Metabolism Genes in M. guilliermondii Strain}

GXDK6 was incubated by using an enrichment technique and GBM liquid medium ( $0.2 \%$ yeast extract, $0.2 \%$ beef extract, $0.5 \%$ polypeptone, $0.6 \%$ sucrose, $\mathrm{pH} 7.0$ ) with $200 \mathrm{rpm}$ and $30^{\circ} \mathrm{C}$ for $8 \mathrm{~h}$. The cells were then collected by centrifugation at $8,000 \mathrm{rpm}$ and $4^{\circ} \mathrm{C}$ for $10 \mathrm{~min}$ and washed repeatedly with $0.1 \mathrm{~mol} / \mathrm{L}$ PBS buffer. The purity of DNA in GXDK6 (extracted by CTAB method) was verified by PCR (Polymerase Chain Reaction) and agarose gel electrophoresis, and then the ITS gene was amplified by PCR (Mo et al., 2021). The ITS sequencing data of GXDK6 were deposited to the National Microbiology Data Center database (http://nmdc.cn) under the Accession Number of NMDCN000022O. The whole-genome sequencing and analysis of GXDK6 were performed by the BGI Genomics Co., Ltd (Shenzhen, China). An online software FastQC (http://www. bioinformatics.babraham.ac.uk/projects/fastqc) was used for the quality control of the second-generation sequencing downtime data. To determine the percentage of single-copy genes in the total single-copy genes, an online software BUSCO (http://busco. ezlab.org, v3.0.2) was conducted to complete the sequence comparison of the genome sequences. The genes related to lipid metabolism in GXDK6 were blasted and analyzed by whole genome method. The pathway enrichment analysis of genes regulating lipid metabolism was annotated according to the KEGG database.

\section{Transcriptome Analysis of $M$. guilliermondii Strain}

GXDK6 was incubated for $16 \mathrm{~h}$ at 0,5 , and $10 \% \mathrm{NaCl}$, respectively. The cells were then collected by centrifugation at $12,000 \mathrm{rpm}$ for $10 \mathrm{~min}$. Total RNA was extracted using Trizol method (Villa-Rodríguez et al., 2018) (Trizol reagent kit, Invitrogen, Carlsbad, CA, United States). RNA quality was assessed on an Agilent 2,100 Bioanalyzer (Agilent Technologies, Palo Alto, CA, United States) and checked using rnase free agarose gel electrophoresis. After total RNA was extracted, eukaryotic mRNA was enriched by Oligo (dT) beads, while prokaryotic mRNA was enriched by removing rRNA by Ribo-ZeroTM Magnetic Kit (Epicentre, Madison, WI, United States). Then the enriched mRNA was fragmented into short fragments using fragmentation buffer and reverse transcripted into cDNA with random primers. Second-strand cDNA were synthesized by DNA polymerase $\mathrm{I}$, rnase $\mathrm{H}, \mathrm{dNTP}$ and buffer. Then the cDNA fragments were purified with QiaQuick PCR extraction kit (Qiagen, Venlo, Netherlands), end repaired, poly(A) added, and ligated to Illumina sequencing adapters. The ligation products were size selected by agarose gel electrophoresis, PCR amplified, and sequenced using Illumina HiSeq2500 by Gene Denovo Biotechnology Co. (Guangzhou, China). RNAs differential expression analysis was performed by DESeq2 (Love et al., 2014) software between two different groups (and by edgeR between two samples) (Robinson et al., 2010). The genes/ transcripts with the parameter of false discovery rate (FDR) below 0.05 and absolute fold change $\geq 2$ were considered differentially expressed genes/transcripts. All samples for transcriptome sequencing were set up in three parallel.

\section{Proteomic Analysis of M. guilliermondii Strain}

The incubation and cell collection of GXDK6 were consistent with the above method. The proteins in GXDK6 were extracted by SDT Lysis methods (Zhu et al., 2014) and separated on $12.5 \%$ SDS-PAGE gel. Protein bands were visualized by Coomassie Blue R-250 staining. The detergent, DTT and other low-molecularweight components were removed using UA buffer by repeated ultrafiltration (Sartorius, $30 \mathrm{kD}$ ). Then $100 \mu \mathrm{L}$ iodoacetamide (100 mM IAA in UA buffer) was added to block reduced cysteine residues. After washing, the protein suspensions were digested with $4 \mu \mathrm{g}$ trypsin (Promega) in $40 \mu \mathrm{L} 0.1 \mathrm{M}$ TEAB buffer overnight at $37^{\circ} \mathrm{C}$, and the resulting peptides were collected as a filtrate. The peptide content was estimated by UV light spectral density at $280 \mathrm{~nm}$ using an extinctions coefficient of 1.1 

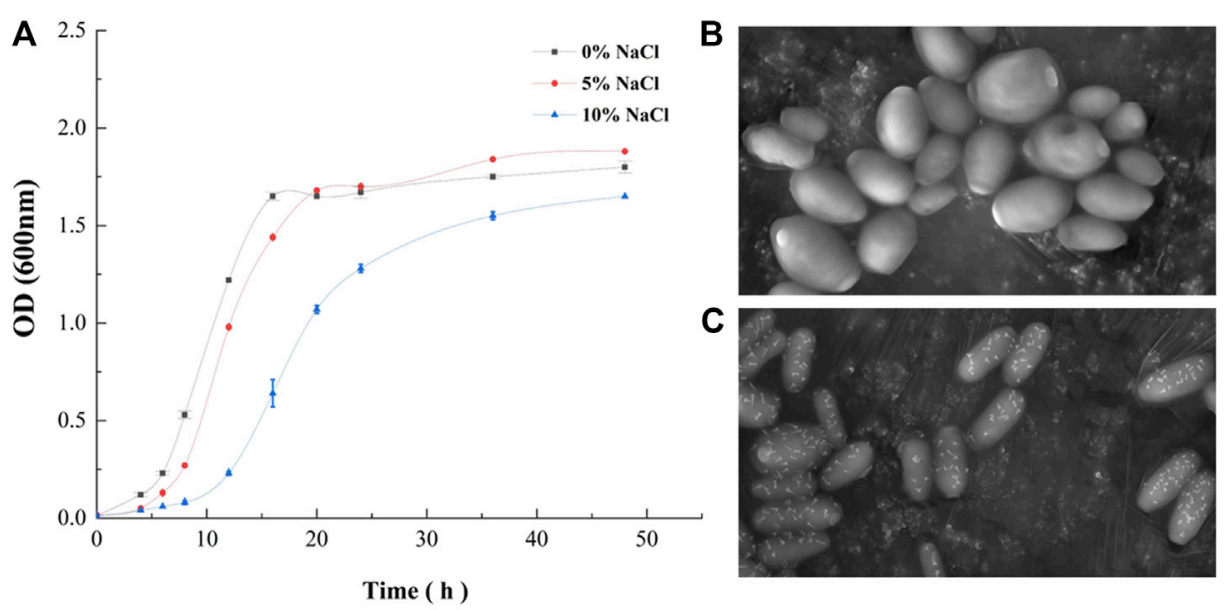

D
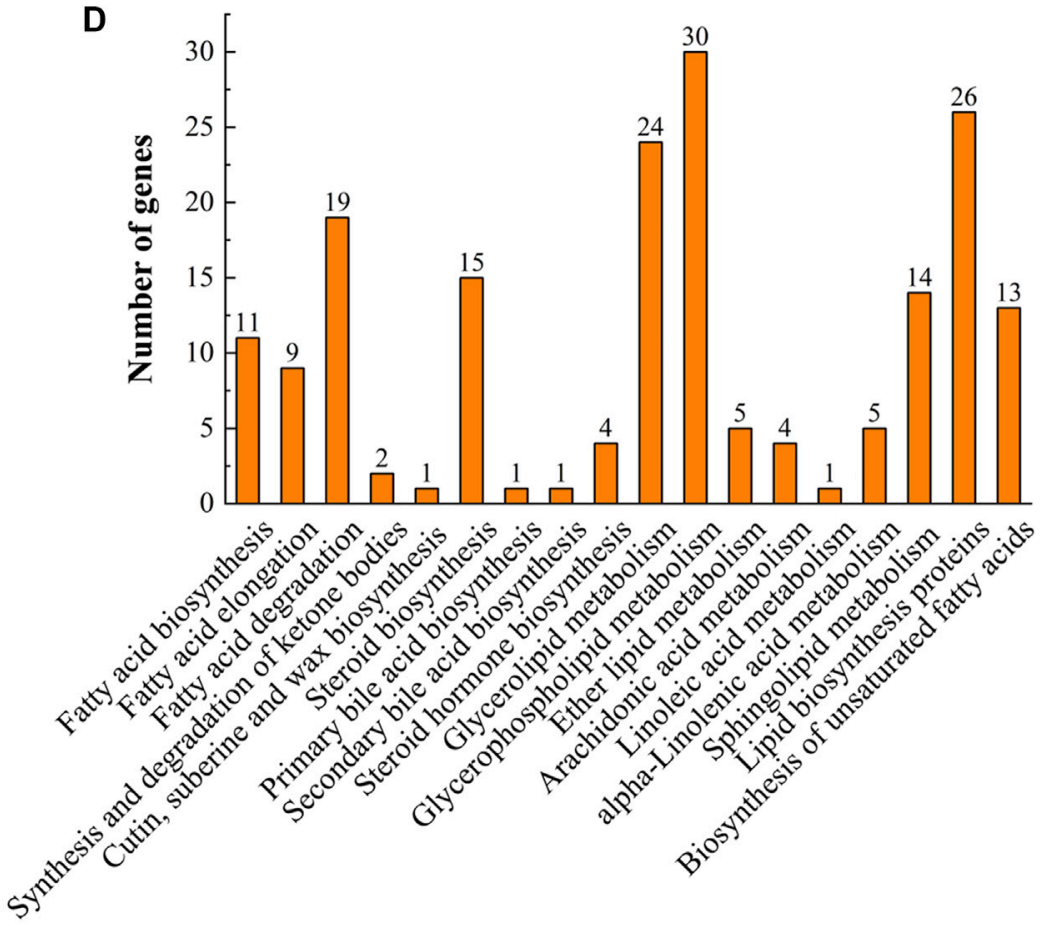

FIGURE 1 | Growth of GXDK6 under NaCl stress. (A) Growth curve of GXDK6 under different NaCl stresses; (B) morphology of GXDK6 under 0\% NaCl; (C) morphology of GXDK6 under $10 \% \mathrm{NaCl}$; (D) Gene enrichment analysis of lipid metabolism.

of $0.1 \%(\mathrm{~g} / \mathrm{L})$ solution. Sequencing of the extracted proteins was conducted by Gene Denovo Biotechnology Co. (Guangzhou, China) by using a tandem mass tag (TMT)-based quantitative proteomics (Myers et al., 2019). TMT labeled peptides were fractionated by RP chromatography using the Agilent 1,260 infinity II HPLC. LC-MS/MS analysis was performed on a Q Exactive plus mass spectrometer (Thermo Fisher Scientific) that was coupled to Easy nLC (Thermo Fisher Scientific) for 60/ $90 \mathrm{~min}$. MS/MS raw files were processed using MASCOT engine (Matrix Science, London, United Kingdom; version 2.6) embedded into Proteome Discoverer 2.2, and searched against the NCBInr/UniProt database. Proteins with Fold change $>1.2$ and $p$ value (Student's $t$ test) $<0.05$ were considered as differentially expressed proteins.

\section{Fluconazole Resistance Test of $M$. guilliermondii Strain}

GXDK6 was inoculated under 0,5 and $10 \% \mathrm{NaCl}$ stress containing fluconazole $(64 \mu \mathrm{g} / \mathrm{ml})$. The culture conditions under 0,5 and $10 \%$ $\mathrm{NaCl}$ stress without fluconazole were set as the controls. Turbidity method and spread plate method was used to investigate the effect of salt stimulation on the drug resistance ability of GXDK6.

\section{RT-qPCR Verification of Transcriptome Data}

According to transcriptome and whole-genome data, GUT1 (encoding glycerol kinase), $A D H 7$ (encoding NADP-dependent alcohol dehydrogenase 7), GPP1 (encoding glycerol-1-phosphate phosphohydrolase 1) were selected and designed (Primer Premier 5.0 software) for qPCR verification in this work (Supplementary Table 
TABLE 1 | KEGG pathway enrichment analysis of lipid metabolism in GXDK6.
Pathway

Fatty acid biosynthesis

Fatty acid elongation

Fatty acid degradation

Synthesis and degradation of ketone bodies

Cutin, suberine and wax biosynthesis

Steroid biosynthesis

Primary bile acid biosynthesis

Secondary bile acid biosynthesis

Steroid hormone biosynthesis

Glycerolipid metabolism

Glycerophospholipid metabolism

Ether lipid metabolism

Arachidonic acid metabolism

Linoleic acid metabolism

alpha-Linolenic acid metabolism

Sphingolipid metabolism

Lipid biosynthesis proteins

Biosynthesis of unsaturated fatty acids
Number of genes

11

9

19

2

1

15

1

24

30

5

4

1

5

14

26

13
S18) [the internal reference gene refers to Díaz et al. (2013)]. The reaction volume of RT-qPCR was $20 \mu \mathrm{L}$. The mixture was heated to $95^{\circ} \mathrm{C}$ for $2 \mathrm{~min}$, followed by 40 cycles of denaturation at $95^{\circ} \mathrm{C}$ for $15 \mathrm{~s}$, and annealing/extension at $60^{\circ} \mathrm{C}$ (depending on the primers) for $30 \mathrm{~s}$. The cycling temperature was then increased by $0.3^{\circ} \mathrm{C}$ every $10 \mathrm{~s}$ from 63 to $95^{\circ} \mathrm{C}$ to obtain the melting curve. Three sets of parallel trials were set up for each gene. Real-time PCR was carried out using a highthroughput real-time fluorescence quantitative PCR instrument ROCHE 480 (ROCHE, United States). Meanwhile, fluorescence detection was performed on LightCycler 480 Software release 1.5.1.62 SP3, and the number of gene amplification cycles was counted in the Software. Relative quantitation was calculated using the $2^{-\Delta \Delta \mathrm{Ct}}$ method (Livak and Schmittgen, 2001).

\section{Effect of Adding Exogenous Glycerol on the Growth of M. guilliermondii Strain}

Exogenous addition of glycerol was used to verify the transcriptome and proteome results. The salt tolerance of GXDK6 was investigated by adding $120 \mathrm{mg} / \mathrm{L}, 1,200 \mathrm{mg} / \mathrm{L}$ and $12,000 \mathrm{mg} / \mathrm{L}$ of exogenous glycerol to salt-containing medium. The liquid medium without glycerol and containing 0,5 and $10 \%$ $\mathrm{NaCl}$ was also set up as a control. Subsequently, $\mathrm{OD}_{600}$ was detected to evaluate the effect of adding exogenous glycerol on the growth of GXDK6.

\section{Data Analysis}

Experimental data were processed with SPSS 25, Origin 2018, Excel 2019, TBtools, and Diamond software. The relevant query databases were Uniprot, SWISS-MODEL, KEGG, and NCBI. Significant difference was at a $p$-value $<0.05$.

\section{RESULTS AND DISCUSSION}

The Growth Status was Changed When $M$. guilliermondii Strain Perceived Salt Stress

The lag phase of GXDK6 under $5-10 \% \mathrm{NaCl}$ stress was prolonged to $4 \mathrm{~h}$, which was approximately $2 \mathrm{~h}$ longer than that of the controls, indicating that the DNA replication and transcription of GXDK6 were inhibited by $\mathrm{NaCl}$ stress (Figure 1A). Besides, the growth of GXDK6 in log phase was remarkably inhibited by $\mathrm{NaCl}$ stress. With the incubation time at $16 \mathrm{~h}$ as an example, the $\mathrm{OD}_{600}$ of GXDK6 from non- $\mathrm{NaCl}$ stress condition was 1.567; it decreased to 1.281 (decreased by $18.25 \%$ ) under $5 \% \mathrm{NaCl}$ stress and 0.558 under $10 \% \mathrm{NaCl}$ stress (decreased by $64.39 \%$ ), suggesting that the metabolism and protein expression of GXDK6 were transformed by $\mathrm{NaCl}$ stress. However, the $\mathrm{OD}_{600}$ value of GXDK6 in stable phase showed an inconspicuous difference under $0-5 \% \mathrm{NaCl}$ stress $(p>$ $0.05)$, demonstrating that GXDK6 has a strong salt-tolerant ability and may need to take a while to adapt to salt stress. Moreover, the morphology of GXDK6 under $\mathrm{NaCl}$ stress was observed by SEM. The results showed that the cell morphology of GXDK6 was round, smooth, and oval under non-salt stress (Figure 1B). However, it showed a contracted, elongated, or rod-shaped form under $10 \%$ $\mathrm{NaCl}$ stress (Figure 1C), suggesting that the morphological changes in GXDK6 may respond to $\mathrm{NaCl}$ stress due to its regulation of lipid metabolism and membrane permeability (Wang D. et al., 2021). For testing of this hypothesis, the transcriptome and proteome of GXDK6 were further investigated under $\mathrm{NaCl}$ stress.

\section{Genome-Wide Analysis Revealed That Lipid Metabolism-Related Genes Were Significantly Enriched in Pathways Contributing to Salt-Tolerant Survival}

117 genes relevant to the lipid metabolism of GXDK6 were annotated, which accounted for $8.77 \%$ of the total metabolic regulation genes (1,334 genes, provided in Supplementary Table S1; Supplementary Figure S1), indicating that GXDK6 had the ability to produce rich lipids. Particularly, mangrove-associated microorganisms resulted in characterization of almost 1,000 new metabolites, among them, $\sim 850$ derived from fungi, and $\sim 120$ from bacteria (Ancheeva et al., 2018; Blunt et al., 2018). Interestingly, whole-genome analysis found M. guilliermondii strain had abundant genes [e.g., ERG1 encoding squalene monooxygenase, ERG5 encoding cytochrome P450 61, ERG6 encoding sterol 24-C-methyltransferase, ERG9 encoding squalene synthase, ERG11 encoding lanosterol 14-alpha demethylase, and ERG24 encoding delta (14)-sterol reductase] regulating steroid biosynthesis pathway, which regulate the biosynthesis of sterols and terpenoids. Therefore, GXDK6 could have a specific regulatory network to biosynthesize important terpenoids. Previous studies have shown that GXDK6 had the ability to biosynthesize nerol (an acyclic monoterpene), which was widely used in food, cosmetics and pharmaceuticals as the valuable fragrance (Mo et al., 2021), aptly validating the above speculation.

In addition, these genes (e.g., GPD encoding glycerol-3phosphate dehydrogenase, ERG24, and GPP1) were mainly involved in regulating glycerophospholipid metabolism, lipid biosynthesis proteins, glycerolipid metabolism, fatty acid degradation, steroid biosynthesis, sphingolipid metabolism, the biosynthesis of unsaturated fatty acids, fatty acid biosynthesis, and fatty acid elongation (Figure 1D; Table 1). Among them, glycerophospholipid metabolism, glycerolipid metabolism, fatty 

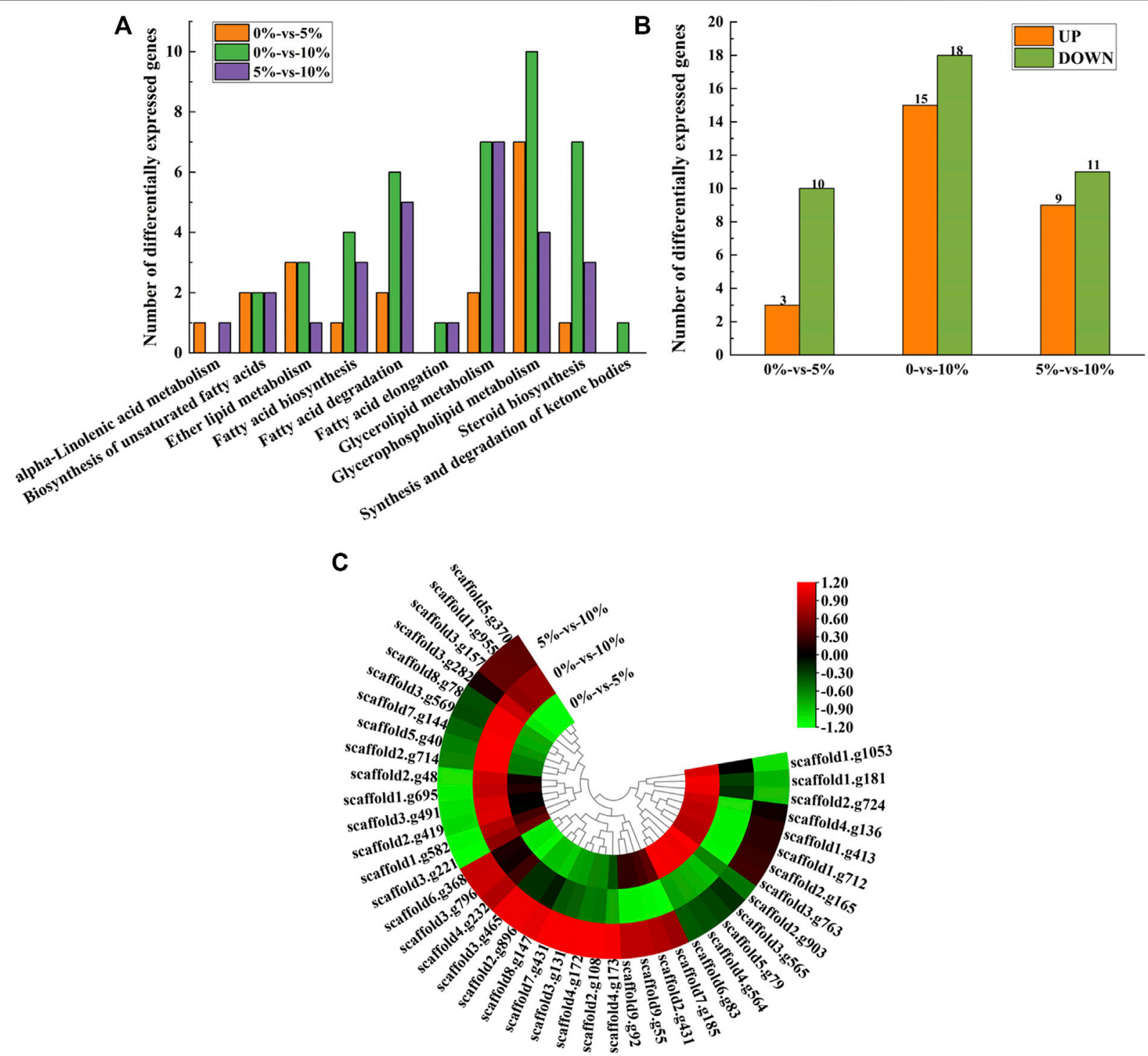

FIGURE 2 | Transcriptome analysis of lipid metabolism. (A) Expression of differentially expressed genes at 0, 5, and 10\% NaCl; (B) Enrichment of pathways under different $\mathrm{NaCl}$ stress; (C) Heatmap of differentially expressed genes.

acid degradation, the biosynthesis of unsaturated fatty acids, fatty acid biosynthesis, and fatty acid elongation played an important role in the defense and survival of microorganisms (Chen et al., 2015). Vigorous lipid metabolism was speculated to have contributed to salt-tolerant survival. Therefore, the transcriptome and proteome of GXDK6 were further studied.

\section{Transcriptome Analysis Revealed That ERG24 Was Significantly Down-Regulated in M. guilliermondii Strain After Perceived Salt Stress}

As shown in Figure 2B, three genes were upregulated and 10 genes were downregulated under $5 \% \mathrm{NaCl}$ stress compared with those under salt-free stress, while 15 genes were upregulated and
18 genes were downregulated under $10 \% \mathrm{NaCl}$ stress. These differentially expressed genes (e.g., GPD, GUT, and GPP) under $10 \% \mathrm{NaCl}$ stress were significantly enriched in glycerophospholipid metabolism (accounting for 30.30\%), glycerolipid metabolism (accounting for 21.21\%), steroid biosynthesis (accounting for $21.21 \%$ ), fatty acid degradation (accounting for $18.18 \%$ ), fatty acid biosynthesis (accounting for $12.12 \%$ ), ether lipid metabolism (accounting for 9.09\%), the biosynthesis of unsaturated fatty acids (accounting for $6.06 \%$ ), fatty acid elongation (accounting for 3.03\%), and synthesis and degradation of ketone bodies (accounting for $3.03 \%$, Figure 2A). Numerous differentially expressed genes (e.g., GPD, GUT, and GPP) that regulate lipid metabolism were significantly enriched in pathways that contributed to salt-tolerant survival (e.g., glycerophospholipid metabolism, 

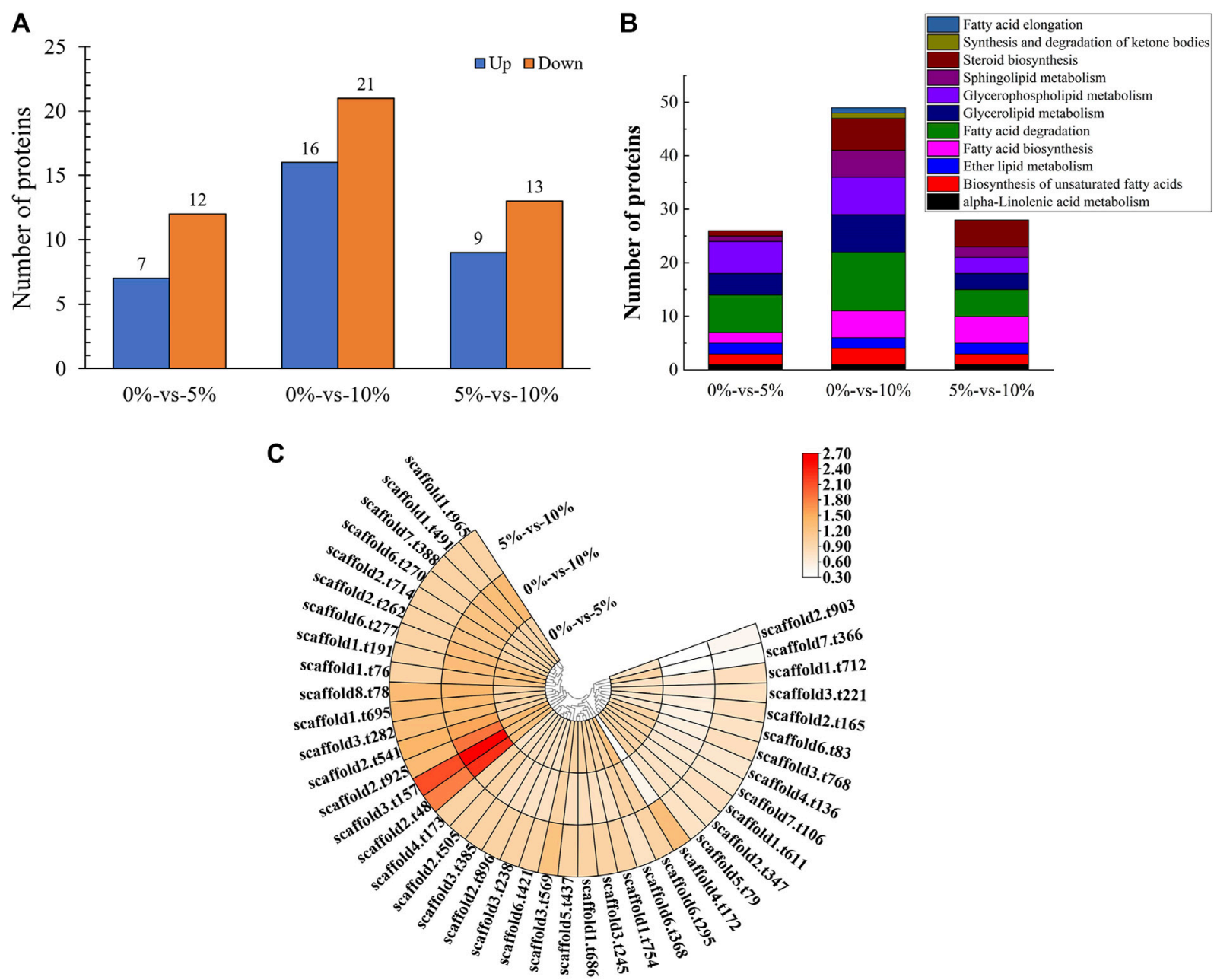

FIGURE 3 | Proteomic analysis of lipid metabolism. (A) Number of differentially expressed proteins under different salt stress; (B) Pathway enrichment of differentially expressed proteins; (C) Differentially expressed proteins under different salt stress.

glycerolipid metabolism, fatty acid degradation) (Xia et al., 2019; Yang et al., 2019) under $10 \% \mathrm{NaCl}$ stress, which meant $M$. guilliermondii strain had good salt resistance, providing a reference for the application in high salt environments (e.g., soy sauce brewing).

With the condition of $10 \% \mathrm{NaCl}$ stress as an example, the most significantly upregulated gene was GPD (upregulated by 4.00 folds) (Figure 2C, provided in Supplementary Table S4). GPD belonged to the HOG-MAPK pathway, which contributed to the timely reception of signals generated by salt stimulation in $M$. guilliermondii strain and induced the expression of targeted regulatory genes in cells, thereby contributing to salt-tolerant survival (Akhtar et al., 2000). According to the analysis of glycerol metabolism, genes (e.g., GUT, ALD5, and GPP) were also significantly up-regulated under $10 \% \quad \mathrm{NaCl}$ stress (Supplementary Figure S2). It would be reasonable to speculate that $M$. guilliermondii strain regulate glycerol metabolism and accumulate to maintain intra- and extracellular osmotic pressure, thereby promoting the survival of GXDK6 under salt stress. Similar results had also been demonstrated by Duskova et al. (2015), Capusoni et al. (2019), and Lee and Levin (2015). We have also confirmed through experiments that exogenous addition of glycerol enhanced the salt tolerance of GXDK6 under stress (Supplementary Figure S3).

However, the most significantly downregulated gene was ERG24 (downregulated by 3.15 folds, provided in Supplementary Table S4). Delta (14)-sterol reductase (regulated by ERG24) is a drug target of fenpropimorph, which is involved in step 2 of the subpathway that synthesizes zymosterol from lanosterol. Due to the significant downregulation of this gene under salt stress, it was speculated that $\mathrm{NaCl}$ stress may change the sensitivity of $M$. guilliermondii strain to drugs. This speculation was indirectly verified by Bhuiyan et al. (2007).

In order to verify the reliability of the transcriptome data, GUT1, GPP1 and ADH7 related to glycerol metabolism were selected for RT-qPCR verification. It can be seen from 


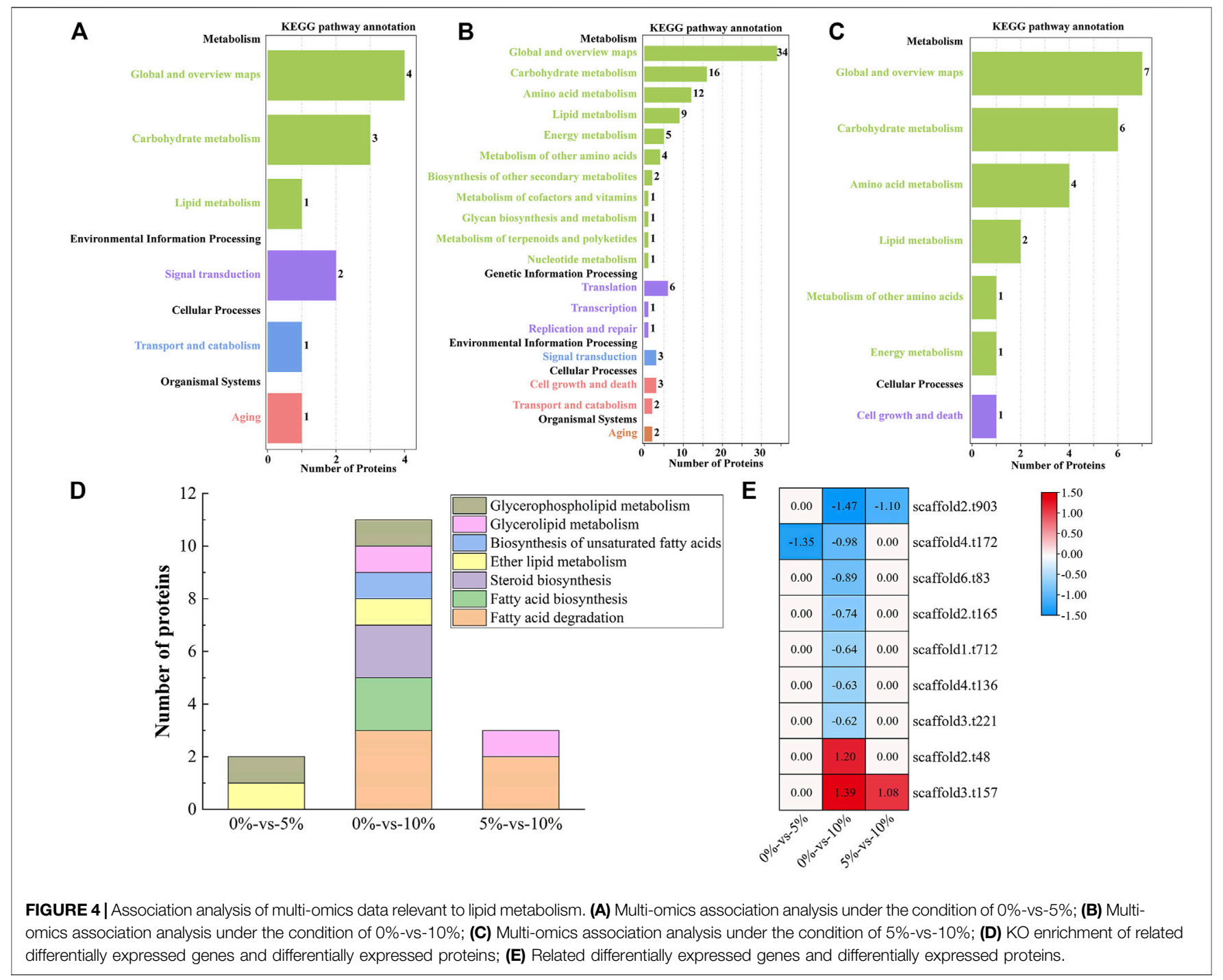

Supplementary Figure S4 that the transcription levels of the three genes verified by RT-qPCR are up-regulated, which is consistent with the results of the transcriptome data, indicating the reliability of the transcriptome data. Furthermore, we analyzed the proteomic data of lipid metabolism in GXDK6.

\section{Proteomic Analysis Revealed That $\mathrm{NaCl}$ Stress Inhibited Spore Germination of $\boldsymbol{M}$. guilliermondii Strain}

16 proteins were significantly upregulated and 21 proteins were significantly downregulated, which were all linked to lipid metabolism in GXDK6 under $10 \% \mathrm{NaCl}$ stress (Figure 3A). These differentially expressed proteins were enriched in fatty acid degradation (accounting for 29.73\%), glycerophospholipid metabolism (accounting for 18.92\%), glycerolipid metabolism (accounting for 18.92\%), steroid biosynthesis (accounting for $16.22 \%$ ), sphingolipid metabolism (accounting for $13.51 \%$ ), fatty acid biosynthesis (accounting for 13.51\%), the biosynthesis of unsaturated fatty acids (accounting for $8.11 \%$ ), ether lipid metabolism (accounting for 5.41\%), alpha-linolenic acid metabolism (accounting for 2.70\%), synthesis and degradation of ketone bodies (accounting for 2.70\%), and fatty acid elongation (accounting for $2.70 \%$, Figure 3B). Among them, the top three significantly upregulated proteins were alcohol dehydrogenase 3 (upregulated by 1.20 folds, promoting the dehydrogenation of aldehydes to produce alcohols, provided in Supplementary Table S11), aldehyde dehydrogenase 5 (upregulated by 1.39 folds, reducing the accumulation of aldehydes, provided in Supplementary Table S11), and 3ketoacyl-thiolase peroxisomal (upregulated by 0.89 folds, a kind of peroxisomal involved in the pathway of fatty acid metabolism). The top three significantly downregulated proteins were NADPH-dependent 1-acyldihydroxyacetone phosphate reductase (downregulated by 0.98 folds; plays a role in cell wall biogenesis, but this effect may be indirect by affecting the activities of cell wall synthesis enzymes), methylsterol 


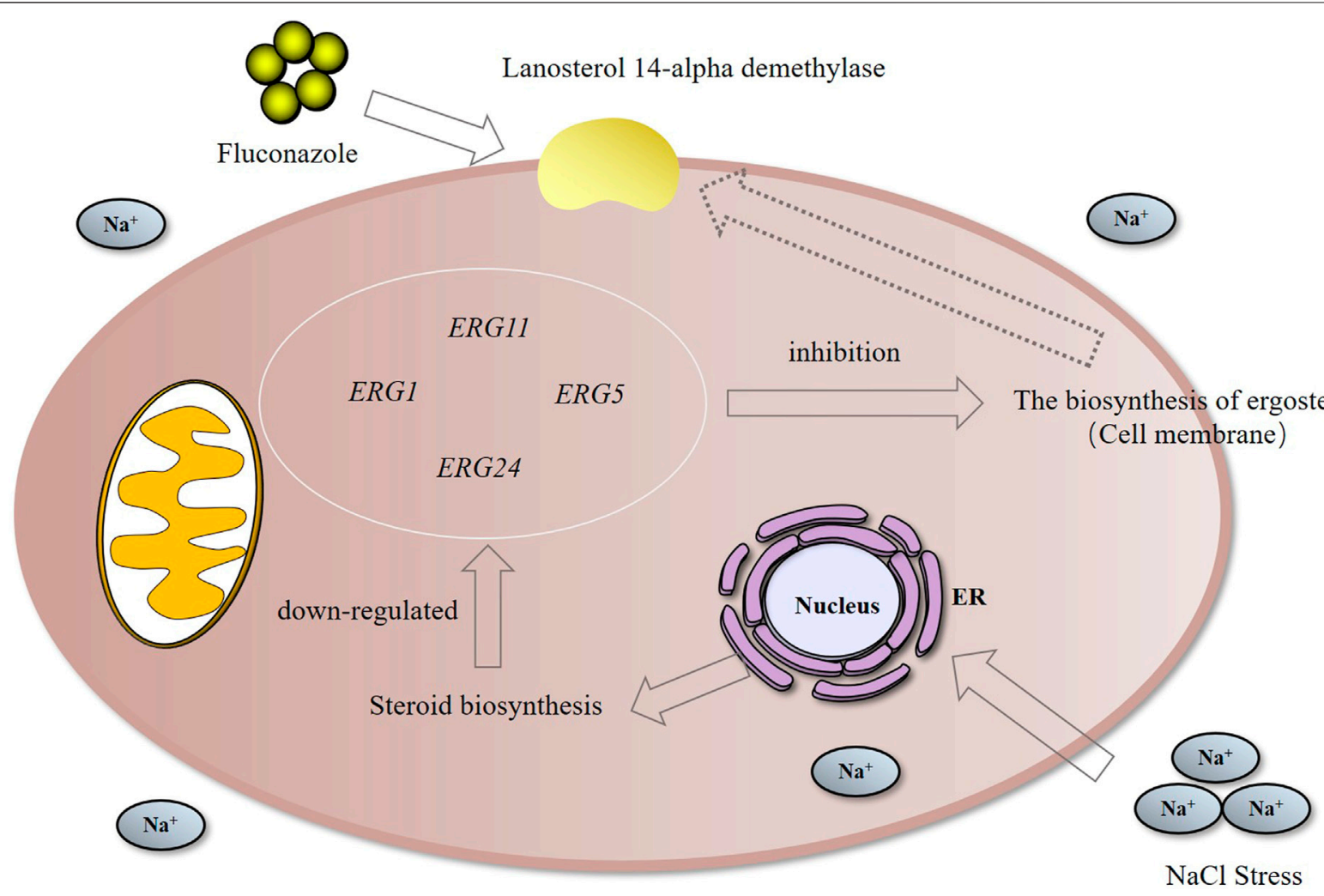

FIGURE 5 | Drug resistance of GXDK6 to fluconazole under $\mathrm{NaCl}$ stress.

monooxygenase (downregulated by 1.43 folds; involved in the pathway of ergosterol biosynthesis, which is a part of steroid metabolism), and alcohol dehydrogenase 2 (downregulated by 1.47 folds; this isozyme preferentially catalyzes the conversion of ethanol to acetaldehyde and acts on various primary unbranched aliphatic alcohols) under $10 \% \mathrm{NaCl}$ (Figure 3C, provided in Supplementary Table S11). Aldehyde dehydrogenase 5 (regulated by $A L D H 5$ ) and alcohol dehydrogenase (regulated by $A D H 2$, and $A D H 3$ ) had functions of protecting cells and maintaining cellular osmotic pressure homeostasis.

Among them, NADPH-dependent 1-acyldihydroxyacetone phosphate reductase (regulatory gene $A Y R 1$, which regulated the budding and division of cell membranes) (Athenstaedt and Daum, 2000) regulated phosphatidic acid biosynthesis. According to significant down-regulated expression of this protein under high salt stress, it was reasonable to speculate that the budding division of the cell membrane was inhibited, thus inhibiting cell proliferation. This finding further revealed the reason for the decrease in the number of cells under $10 \%$ $\mathrm{NaCl}$. The test showed that with the incubation time at $16 \mathrm{~h}$ as an example, the $\mathrm{OD}_{600}$ of GXDK6 decreased by $64.39 \%$ under $10 \% \mathrm{NaCl}$ stress when compared with salt-free stress. The presence of $\mathrm{NaCl}$ during the cultivation process caused a slight decrease in the growth rate. This result was also confirmed by Capusoni et al. (2019) and Petrovic et al. (2002). The analysis of the growth state of M. guilliermondii strain under salt stress could provide a reference for its application in high-salt environments.

In addition, the methylsterol monooxygenase regulates the biosynthesis of ergosterol (Ward et al., 2018), which contributes to maintaining the rigidity of the plasma membrane (Schützhold et al., 2016), and serves as the precursor for manufacturing steroid drugs (Liu et al., 2019). However, the methylsterol monooxygenase (subcellularly located in the endoplasmic reticulum membrane) was significantly down-regulated under $10 \% \mathrm{NaCl}$ stress. It was reasonable to speculate that $M$. guilliermondii strain can regulate the expression of membrane proteins to enhance resistance to stress environments.

\section{Transcriptomic and Proteomic Analysis Revealed That Lanosterol 14-Alpha Demethylase was Down-Regulated in $M$. guilliermondii Strain After Perceived Salt Stress}

One differentially expressed gene (AYR1) and one differentially expressed protein (NADPH-dependent 1-acyldihydroxyacetone phosphate reductase, which could convert acyl and alkyl dihydroxyacetone-phosphate into glycerolipids and ether lipids) were associated in the pathway of lipid metabolism under $5 \% \mathrm{NaCl}$ compared with those under salt-free stress (Figure 4A, provided in Supplementary Table S14), while nine differentially expressed genes and nine differentially 
TABLE 2 | Drug resistance of GXDK6 to fluconazole under $\mathrm{NaCl}$ stress.

\begin{tabular}{llc}
\hline Conditions & Dilution times & Cfu/ml \\
\hline $0 \% \mathrm{NaCl}+0 \mu \mathrm{g} / \mathrm{ml}$ fluconazole & $10^{-6}$ & $2.1^{\star} 10^{8}$ \\
$5 \% \mathrm{NaCl}+0 \mu \mathrm{g} / \mathrm{ml}$ fluconazole & $10^{-6}$ & $1.0^{\star} 10^{7}$ \\
$10 \% \mathrm{NaCl}+0 \mu \mathrm{g} / \mathrm{ml}$ fluconazole & $10^{-5}$ & $9.0^{\star} 10^{5}$ \\
$0 \% \mathrm{NaCl}+64 \mu \mathrm{g} / \mathrm{ml}$ fluconazole & $10^{-5}$ & $1.0^{\star} 10^{6}$ \\
$5 \% \mathrm{NaCl}+64 \mu \mathrm{g} / \mathrm{ml}$ fluconazole & $10^{-3}$ & $1.1^{\star} 10^{5}$ \\
$10 \% \mathrm{NaCl}+64 \mu \mathrm{g} / \mathrm{ml}$ fluconazole & $10^{-2}$ & $2.3^{\star} 10^{4}$ \\
\hline
\end{tabular}

expressed proteins were associated under 10\% $\mathrm{NaCl}$ (Figures 4B,E, provided in Supplementary Table S15). When GXDK6 was under $10 \% \mathrm{NaCl}$, two differentially expressed genes and two differentially expressed protein were associated in lipid metabolism compared with those under $5 \% \mathrm{NaCl}$ stress (Figure 4C, provided in Supplementary Table S16). With the condition of $10 \% \mathrm{NaCl}$ stress as an example, the related genes and proteins were mainly enriched in fatty acid degradation (accounting for 33.33\%), fatty acid biosynthesis (accounting for 22.22\%), steroid biosynthesis (accounting for 22.22\%), ether lipid metabolism (accounting for 11.11\%), the biosynthesis of unsaturated fatty acids (accounting for $11.11 \%$ ), glycerolipid metabolism (accounting for 11.11\%), and glycerophospholipid metabolism (accounting for $11.11 \%$, Figure 4D). These differentially expressed proteins were alcohol dehydrogenase 2 (downregulated by 1.47 folds), NADPH-dependent 1-acyl dihydroxyacetone phosphate reductase (downregulated by 0.98 folds), squalene monooxygenase (downregulated by 0.89 folds), fatty acid synthase alpha subunit (downregulated by 0.74 folds), acetylCoA carboxylase (downregulated by 0.64 folds), lanosterol 14alpha demethylase (downregulated by 0.63 folds), delta (12)fatty-acid desaturase (downregulated by 0.62 folds), alcohol dehydrogenase 3 (upregulated by 1.20 folds), and aldehyde dehydrogenase (upregulated by 1.39 folds).

Squalene monooxygenase was the key rate-limiting enzyme for the synthesis of sterols and triterpenoids (Han et al., 2020). The expression of this enzyme was downregulated under $10 \%$ $\mathrm{NaCl}$ stress, indicating that the synthesis of sterol substances was changed under salt stress. Lanosterol 14-alpha demethylase was a key enzyme in ergosterol biosynthesis. When ergosterol synthesis was blocked, the cell membranes were damaged, thus making antifungal drugs, such as azoles, work (Kauffman and Carver, 1997; Sagatova et al., 2015). Studies have found that the protein was significantly differentially expressed under salt stress. It was speculated that the killing effect of antifungal drugs would be affected by $\mathrm{NaCl}$ stress. Therefore, we explored the changes of resistance in GXDK6 under salt stress.

\section{$\mathrm{NaCl}$ Stress Enhanced the Killing Effect of Fluconazole on $M$. guilliermondii Strain}

As shown in Supplementary Table S4, the differentially expressed genes were ERG1 (downregulated by 2.34 folds, encoding squalene monooxygenase), ERG5 (downregulated by 1.09 folds, encoding squalene monooxygenase), ERG11 (downregulated by -1.17 folds, encoding lanosterol 14-alpha demethylase), and ERG24 [downregulated by 3.15 folds, encoding delta (14)-sterol reductase]. The above genes were mainly involved in the biosynthesis of ergosterol (Konecna et al., 2016; Guo et al.,2018). The critical role of ergosterol in maintaining cell membrane integrity and its uniqueness in fungi make it a highly attractive antifungal drug target (Li Y. et al., 2021). Previous studies had shown that ERG24 was an antifungal target site for azole drugs (Jia et al., 2002). Flowers et al. (2015) found that single mutations in ERG11 (an important drug target) contributed to azole resistance in Candida albicans. In this study, these genes were significantly downregulated under salt stress. Therefore, we speculated that ergosterol, as a target of antifungal drugs, its synthesis pathway was inhibited under salt stress, leading to improve killing effect of fluconazole against $M$. guilliermondii strain (Long et al., 2017). This speculation was also confirmed in the present work, and the results were shown in Figure 5. The $\mathrm{OD}_{600}$ of GXDK6 under $10 \% \mathrm{NaCl}$ and contained $64 \mu \mathrm{g} / \mathrm{ml}$ fluconazole was 0.319 . However, it decreased to 0.124 under $64 \mu \mathrm{g} / \mathrm{ml}$ fluconazole, which was $61.13 \%$ lower than the condition of salt-free but contained $64 \mu \mathrm{g} / \mathrm{ml}$ fluconazole (provided in Supplementary Figure S5). Moreover, the number of colonies of GXDK6 under $10 \% \mathrm{NaCl}$ and without fluconazole were $9.0^{\star} 10^{5} \mathrm{cfu} / \mathrm{ml}$ (provided in Supplementary Figure S6). However, it decreased to $2.3^{\star} 10^{4} \mathrm{cfu} / \mathrm{ml}$ under $64 \mu \mathrm{g} /$ $\mathrm{ml}$ fluconazole, which was $97.70 \%$ lower than the condition of salt-free but contained $64 \mu \mathrm{g} / \mathrm{ml}$ fluconazole (number of colonies = $1.0^{\star} 10^{6} \mathrm{cfu} / \mathrm{ml}$, Table 2). It was considered that the genes regulating synthesis of ergosterol were down-regulated under salt stress, which lead to down-regulation of the corresponding proteins expression, thereby weakening the synthesis of ergosterol. The results demonstrated that salt stress promoted the fungicidal effect of fluconazole, which provided a new approach to enhance the killing effect of fluconazole on fungi.

\section{CONCLUSION}

The regulation mechanism of lipid metabolism in M. guilliermondii GXDK6 was revealed by integrative omics technology. The key genes and proteins that regulated steroid biosynthesis and glycerolipid metabolism under salt stress were identified. High-concentration salt stress could change the biosynthesis of ergosterol and inhibited cell proliferation. Further results demonstrated that the killing effect of fluconazole on M. guilliermondii GXDK6 was significantly enhanced under salt stimulation. Final results showed that salt stress disturbed lipid metabolism, which reduced the expression of antifungal drugs targets, thus making $M$. guilliermondii strain more sensitive to fluconazole. This work revealed the regulatory changes of lipid metabolism in M. guilliermondii strain under salt stress, which also provided a new strategy to enhance the fungicidal effect of antifungal drugs.

\section{DATA AVAILABILITY STATEMENT}

The datasets presented in this study can be found in online repositories. The names of the repository/repositories and accession number(s) can be found in the article/Supplementary Material. 


\section{AUTHOR CONTRIBUTIONS}

CJ designed research. HS conducted the experiments and wrote the manuscript. XC conducted the experiments and revised the manuscript. HB and DM arranged and analyzed the experimental data. XM and SH provided the technical support. BY provided theoretical direction of multi-omics research. GS corrected the language errors of the manuscript. All the authors read and approved the final manuscript.

\section{FUNDING}

This research was supported by the National Natural Science Foundation of China (Grant No. 31760437), Science and Technology Basic Resources Investigation Program of China

\section{REFERENCES}

Akhtar, N., Påhlman, A. K., Larsson, K., Corbett, A. H., and Adler, L. (2000). SGD1 Encodes an Essential Nuclear Protein of Saccharomyces cerevisiae that Affects Expression of the GPD1 Gene for Glycerol 3-phosphate Dehydrogenase. FEBS Lett. 483 (2-3), 87-92. doi:10.1016/s0014-5793(00)02087-1

Ancheeva, E., Daletos, G., and Proksch, P. (2018). Lead Compounds from Mangrove-Associated Microorganisms. Mar. Drugs 16 (9), 319. doi:10.3390/ md16090319

Athenstaedt, K., and Daum, G. (2000). 1-Acyldihydroxyacetone-phosphate Reductase (Ayrlp) of the Yeast Saccharomyces cerevisiae Encoded by the Open reading Frame YIL124w Is a Major Component of Lipid Particles. J. Biol. Chem. 275 (1), 235-240. doi:10.1074/jbc.275.1.235

Bhuiyan, M. S. A., Eckstein, J., Barbuch, R., and Bard, M. (2007). Synthetically Lethal Interactions Involving Loss of the Yeast ERG24: the Sterol C-14 Reductase Gene. Lipids 42 (1), 69-76. doi:10.1007/s11745-006-1001-4

Blunt, J. W., Carroll, A. R., Copp, B. R., Davis, R. A., Keyzers, R. A., and Prinsep, M. R. (2018). Marine Natural Products. Nat. Prod. Rep. 35 (1), 8-53. doi:10.1039/ c7np00052a

Capusoni, C., Arioli, S., Donzella, S., Guidi, B., Serra, I., and Compagno, C. (2019). Hyper-osmotic Stress Elicits Membrane Depolarization and Decreased Permeability in Halotolerant marine Debaryomyces Hansenii Strains and in Saccharomyces cerevisiae. Front. Microbiol. 10, 64. doi:10.3389/ fmicb.2019.00064

Casanovas, A., Sprenger, R. R., Tarasov, K., Ruckerbauer, D. E., Hannibal-Bach, H. K., Zanghellini, J., et al. (2015). Quantitative Analysis of Proteome and Lipidome Dynamics Reveals Functional Regulation of Global Lipid Metabolism. Chem. Biol. 22 (3), 412-425. doi:10.1016/j.chembiol.2015.02.007

Chen, K., Li, E., Xu, Z., Li, T., Xu, C., Qin, J. G., et al. (2015). Comparative Transcriptome Analysis in the Hepatopancreas Tissue of pacific white Shrimp litopenaeus Vannamei Fed Different Lipid Sources at Low Salinity. PLoS One 10 (12), e0144889. doi:10.1371/journal.pone.0144889

Díaz, C., Molina, A. M., Nähring, J., and Fischer, R. (2013). Characterization and Dynamic Behavior of Wild Yeast during Spontaneous Wine Fermentation in Steel Tanks and Amphorae. Biomed. Res. Int. 2013, 540465. doi:10.1155/2013/ 540465

Duskova, M., Borovikova, D., Herynkova, P., Rapoport, A., and Sychrova, H. (2015). The Role of Glycerol Transporters in Yeast Cells in Various Physiological and Stress Conditions. FEMS Microbiol. Lett. 362 (3), 1-8. doi:10.1093/femsle/fnu041

Fan, J., Li, X., Issop, L., Culty, M., and Papadopoulos, V. (2016). ACBD2/ECI2mediated Peroxisome-Mitochondria Interactions in Leydig Cell Steroid Biosynthesis. Mol. Endocrinol. 30 (7), 763-782. doi:10.1210/me.2016-1008

Ferreira, R., Teixeira, P. G., Siewers, V., and Nielsen, J. (2018). Redirection of Lipid Flux toward Phospholipids in Yeast Increases Fatty Acid Turnover and
(Grant No. 2017FY100704), the Funding Project of Chinese Central Government Guiding to the Guangxi Local Science and Technology Development (Grant No. GUIKEZY21195021), Natural Science Fund for Distinguished Young Scholars of Guangxi Zhuang Autonomous Region of China (Grant No. 2019GXNSFFA245011), China-ASEAN International Innovative Center for Health Industry of Traditional Chinese Medicine (AD20297142), and the Innovation Project of Guangxi Graduate Education (Grant No. YCBZ2021012 and YCSW2021064).

\section{SUPPLEMENTARY MATERIAL}

The Supplementary Material for this article can be found online at: https:/www.frontiersin.org/articles/10.3389/fgene.2021.798535/ full\#supplementary-material

Secretion. Proc. Natl. Acad. Sci. U S A. 115 (6), 1262-1267. doi:10.1073/ pnas. 1715282115

Flowers, S. A., Colón, B., Whaley, S. G., Schuler, M. A., and Rogers, P. D. (2015). Contribution of Clinically Derived Mutations in ERG11 to Azole Resistance in Candida Albicans. Antimicrob. Agents Chemother. 59 (1), 450-460. doi:10.1128/AAC.03470-14

Galafassi, S., Toscano, M., Vigentini, I., Zambelli, P., Simonetti, P., Foschino, R., et al. (2015). Cold Exposure Affects Carbohydrates and Lipid Metabolism, and Induces Hoglp Phosphorylation in Dekkera Bruxellensis Strain CBS 2499. Antonie Van Leeuwenhoek 107 (5), 1145-1153. doi:10.1007/s10482015-0406-6

Guo, Z. P., Khoomrung, S., Nielsen, J., and Olsson, L. (2018). Changes in Lipid Metabolism Convey Acid Tolerance in Saccharomyces cerevisiae. Biotechnol. Biofuels 11, 297. doi:10.1186/s13068-018-1295-5

Han, J. Y., Jo, H. J., and Choi, Y. E. (2020). Overexpression of the Squalene Epoxidase Gene (PgSE1) Resulted in Enhanced Production of Ginsenosides and Phytosterols in Transgenic Ginseng. Plant Biotechnol. Rep. 14, 673-682. doi:10.1007/s11816-020-00643-4

Jia, N., Arthington-Skaggs, B., Lee, W., Pierson, C. A., Lees, N. D., Eckstein, J., et al. (2002). Candida Albicans Sterol C-14 Reductase, Encoded by the ERG24 Gene, as a Potential Antifungal Target Site. Antimicrob. Agents Chemother. 46 (4), 947-957. doi:10.1128/AAC.46.4.947-957.2002

Kauffman, C. A., and Carver, P. L. (1997). Use of Azoles for Systemic Antifungal Therapy. Adv. Pharmacol. 39, 143-189. doi:10.1016/s1054-3589(08)60071-x

Klug, L., and Daum, G. (2014). Yeast Lipid Metabolism at a Glance. FEMS Yeast Res. 14 (3), 369-388. doi:10.1111/1567-1364.12141

Konecna, A., Toth Hervay, N., Valachovic, M., and Gbelska, Y. (2016). ERG6 Gene Deletion Modifies Kluyveromyces Lactis Susceptibility to Various Growth Inhibitors. Yeast 33 (12), 621-632. doi:10.1002/yea.3212

Kraft, M. L. (2017). Sphingolipid Organization in the Plasma Membrane and the Mechanisms that Influence it. Front Cel Dev Biol 4, 154. doi:10.3389/ fcell.2016.00154

Lee, J., and Levin, D. E. (2015). Rgc2 Regulator of Glycerol Channel Fps1 Functions as a Homo- and Heterodimer with Rgcl. Eukaryot. Cel 14 (7), 719-725. doi:10.1128/EC.00073-15

Li, C., Zhao, D., Yan, J., Zhang, N., and Li, B. (2021). Metabolomics Integrated with Transcriptomics: Assessing the central Metabolism of marine Red Yeast Sporobolomyces Pararoseus under Salinity Stress. Arch. Microbiol. 203 (3), 889-899. doi:10.1007/s00203-020-02082-9

Li, Y., Dai, M., Zhang, Y., and Lu, L. (2021). The Sterol C-14 Reductase Erg24 Is Responsible for Ergosterol Biosynthesis and Ion Homeostasis in Aspergillus fumigatus. Appl. Microbiol. Biotechnol. 105 (3), 1253-1268. doi:10.1007/ s00253-021-11104-5

Liu, J. F., Xia, J. J., Nie, K. L., Wang, F., and Deng, L. (2019). Outline of the Biosynthesis and Regulation of Ergosterol in Yeast. World J. Microbiol. Biotechnol. 35 (7), 98. doi:10.1007/s11274-019-2673-2 
Livak, K. J., and Schmittgen, T. D. (2001). Analysis of Relative Gene Expression Data Using Real-Time Quantitative PCR and the 2(-Delta Delta C(T)) Method. Methods 25 (4), 402-408. doi:10.1006/meth.2001.1262

Long, N., Xu, X., Zeng, Q., Sang, H., and Lu, L. (2017). Erg4A and Erg4B Are Qequired for Conidiation and Azole Resistance via Regulation of Ergosterol Biosynthesis in Aspergillus fumigatus. Appl. Environ. Microbiol. 83 (4), e02924-16. doi:10.1128/AEM.02924-16

Love, M. I., Huber, W., and Anders, S. (2014). Moderated Estimation of Fold Change and Dispersion for RNA-Seq Data with DESeq2. Genome Biol. 15 (12), 550. doi:10.1186/s13059-014-0550-8

Lu, H., Chen, H., Tang, X., Yang, Q., Zhang, H., Chen, Y. Q., et al. (2021). Metabolomics Analysis Reveals the Role of Oxygen Control in the Nitrogen Limitation Induced Lipid Accumulation in Mortierella Alpina. J. Biotechnol. 325, 325-333. doi:10.1016/j.jbiotec.2020.10.004

Mo, X., Cai, X., Hui, Q., Sun, H., Yu, R., Bu, R., et al. (2021). Whole Genome Sequencing and Metabolomics Analyses Reveal the Biosynthesis of Nerol in a Multi-Stress-Tolerant Meyerozyma Guilliermondii GXDK6. Microb. Cel Fact 20 (1), 4. doi:10.1186/s12934-020-01490-2

Myers, S. A., Klaeger, S., Satpathy, S., Viner, R., Choi, J., Rogers, J., et al. (2019). Evaluation of Advanced Precursor Determination for Tandem Mass Tag (TMT)-based Quantitative Proteomics across Instrument Platforms. J. Proteome Res. 18 (1), 542-547. doi:10.1021/acs.jproteome.8b00611

Nebauer, R., Birner-Grünberger, R., and Daum, G. (2004). 3 Biogenesis and Cellular Dynamics of Glycerophospholipids in the Yeast Saccharomyces cerevisiae. Top. Curr. Genet. 6, 125-168. doi:10.1007/978-3-540-40999-1_4

Petrovic, U., Gunde-Cimerman, N., and Plemenitas, A. (2002). Cellular Responses to Environmental Salinity in the Halophilic Black Yeast Hortaea Werneckii. Mol. Microbiol. 45 (3), 665-672. doi:10.1046/j.13652958.2002.03021.x

Qi, Y., Liu, H., Yu, J., Chen, X., and Liu, L. (2017). Med15B Regulates Acid Stress Response and Tolerance in Candida Glabrata by Altering Membrane Lipid Composition. Appl. Environ. Microbiol. 83 (18), e01128-17. doi:10.1128/ AEM.01128-17

Randez-Gil, F., Córcoles-Saez, I., Estruch, F., and Prieto, J. A. (2018). Lipid Metabolism Regulation and its Relationship with Cold Stress Response in Yeast. Cryobiology 85, 127. doi:10.1016/j.cryobiol.2018.10.041

Robinson, M. D., McCarthy, D. J., and Smyth, G. K. (2010). edgeR: a Bioconductor Package for Differential Expression Analysis of Digital Gene Expression Data. Bioinformatics 26 (1), 139-140. doi:10.1093/bioinformatics/btp616

Ross, J. A., Maingay, J. P., Fearon, K. C., Sangster, K., and Powell, J. J. (2003). Eicosapentaenoic Acid Perturbs Signalling via the NFkappaB Transcriptional Pathway in Pancreatic Tumour Cells. Int. J. Oncol. 23 (6), 1733-1738. doi:10.3892/ijo.23.6.1733

Sagatova, A. A., Keniya, M. V., Wilson, R. K., Monk, B. C., and Tyndall, J. D. (2015). Structural Insights into Binding of the Antifungal Drug Fluconazole to Saccharomyces cerevisiae Lanosterol 14a-Demethylase. Antimicrob. Agents Chemother. 59 (8), 4982-4989. doi:10.1128/AAC.00925-15

Schützhold, V., Hahn, J., Tummler, K., and Klipp, E. (2016). Computational Modeling of Lipid Metabolism in Yeast. Front. Mol. Biosci. 3, 57. doi:10.3389/fmolb.2016.00057

Tian, Y., Xia, Z., Li, M., Zhang, G., Cui, H., Li, B., et al. (2019). The Relationship between Microwave Radiation Injury and Abnormal Lipid Metabolism. Chem. Phys. Lipids 225, 104802. doi:10.1016/j.chemphyslip.2019.104802

Villa-Rodríguez, E., Ibarra-Gámez, C., and de Los Santos-Villalobos, S. (2018). Extraction of High-Quality RNA from Bacillus Subtilis with a Lysozyme Pre- treatment Followed by the Trizol Method. J. Microbiol. Methods 147, 14-16. doi:10.1016/j.mimet.2018.02.011

Wang, D., Zhang, M., Huang, J., Zhou, R., Jin, Y., Zhao, D., et al. (2021). Heat Preadaptation Improved the Ability of Zygosaccharomyces Rouxii to Salt Stress: a Combined Physiological and Transcriptomic Analysis. Appl. Microbiol. Biotechnol. 105 (1), 259-270. doi:10.1007/s00253-020-11005-Z

Wang, G., Li, D., Miao, Z., Zhang, S., Liang, W., and Liu, L. (2018). Comparative Transcriptome Analysis Reveals Multiple Functions for Mhylp in Lipid Biosynthesis in the Oleaginous Yeast Yarrowia Lipolytica. Biochim. Biophys. Acta Mol. Cel Biol Lipids 1863 (1), 81-90. doi:10.1016/ j.bbalip.2017.10.003

Wang, S., Tang, K., Lu, Y., Tian, Z., Huang, Z., Wang, M., et al. (2021). Revealing the Role of Glycerophospholipid Metabolism in Asthma through Plasma Lipidomics. Clin. Chim. Acta 513, 34-42. doi:10.1016/j.cca.2020.11.026

Ward, D. M., Chen, O. S., Li, L., Kaplan, J., Bhuiyan, S. A., Natarajan, S. K., et al. (2018). Altered Sterol Metabolism in Budding Yeast Affects Mitochondrial Iron-Sulfur (Fe-S) Cluster Synthesis. J. Biol. Chem. 293 (27), 10782-10795. doi:10.1074/jbc.RA118.001781

Xia, Z., Zhou, X., Li, J., Li, L., Ma, Y., Wu, Y., et al. (2019). Multiple-omics Techniques Reveal the Role of Glycerophospholipid Metabolic Pathway in the Response of Saccharomyces cerevisiae against Hypoxic Stress. Front. Microbiol. 10, 1398. doi:10.3389/fmicb.2019.01398

$\mathrm{Xu}, \mathrm{S}$., and Li, Y. (2020). Yeast as a Promising Heterologous Host for Steroid Bioproduction. J. Ind. Microbiol. Biotechnol. 47 (9-10), 829-843. doi:10.1007/ s10295-020-02291-7

Yang, H. L., Liao, Y. Y., Zhang, J., and Wang, X. L. (2019). Comparative Transcriptome Analysis of Salt Tolerance Mechanism of Meyerozyma Guilliermondii W2 under $\mathrm{NaCl}$ Stress. 3 Biotech. 9 (7), 286. doi:10.1007/s13205-019-1817-2

Zhang, P., and Reue, K. (2017). Lipin Proteins and Glycerolipid Metabolism: Roles at the ER Membrane and beyond. Biochim. Biophys. Acta Biomembr 1859 (9 Pt B), 1583-1595. doi:10.1016/j.bbamem.2017.04.007

Zhou, Y. J., Hu, Y., Zhu, Z., Siewers, V., and Nielsen, J. (2018). Engineering 1alkene Biosynthesis and Secretion by Dynamic Regulation in Yeast. ACS Synth. Biol. 7 (2), 584-590. doi:10.1021/acssynbio.7b00338

Zhu, Y., Xu, H., Chen, H., Xie, J., Shi, M., Shen, B., et al. (2014). Proteomic Analysis of Solid Pseudopapillary Tumor of the Pancreas Reveals Dysfunction of the Endoplasmic Reticulum Protein Processing Pathway. Mol. Cel Proteomics 13 (10), 2593-2603. doi:10.1074/mcp.M114.038786

Conflict of Interest: The authors declare that the research was conducted in the absence of any commercial or financial relationships that could be construed as a potential conflict of interest.

Publisher's Note: All claims expressed in this article are solely those of the authors and do not necessarily represent those of their affiliated organizations, or those of the publisher, the editors and the reviewers. Any product that may be evaluated in this article, or claim that may be made by its manufacturer, is not guaranteed or endorsed by the publisher.

Copyright $\odot 2022$ Sun, Cai, Yan, Bai, Meng, Mo, He, Su and Jiang. This is an openaccess article distributed under the terms of the Creative Commons Attribution License (CC BY). The use, distribution or reproduction in other forums is permitted, provided the original author(s) and the copyright owner(s) are credited and that the original publication in this journal is cited, in accordance with accepted academic practice. No use, distribution or reproduction is permitted which does not comply with these terms. 